\title{
Article
}

\section{Comparative Transcriptome Analyses of metastatic and non-metastatic colorectal cancer cells delineate differential mechanisms of CHOP upregulation in response to ONC201 treatment.}

Ashraf Al Madhoun ${ }^{1,2^{*}}$, Dania Haddad ${ }^{1}$, Mustafa Al Tarrah ${ }^{1}$, Sindhu Jacob ${ }^{1}$, Waleed Al-Ali ${ }^{3}$, Rasheeba Nizam¹, Lavina Miranda², Fatema Al-Rashed ${ }^{4}$, Sardar Sindhu'2,4, Rasheed Ahmad ${ }^{4}$, Milad S. Bitar ${ }^{3}$, and Fahd Al-Mulla ${ }^{*}$

1 Department of Genetics and Bioinformatics, Dasman Diabetes Institute, Dasman, 15462, Kuwait.

2 Department of Animal and Imaging Core Facilities, Dasman Diabetes Institute, Dasman, 15462, Kuwait.

3 Department of Pharmacology and Toxicology, Faculty of Medicine, Kuwait University, 046302 Jabriya, Kuwait.

4 Department of Immunology an Microbiology, Dasman Diabetes Institute, Dasman, 15462, Kuwait.

* Correspondence: fahd@al-mulla.org (F.A.-M.); ashraf.madhoun@dasmaninstitute.org (A.A.M.); Tel.: +965-2224-2999 (ext. 2805) (A.A.M.); +965-6777-1040 (F.A-M.)

\section{ABSTRACT}

The imipramine ONC201 exerts a novel anti-proliferative activity over a wide spectrum of cancer cell types. ONC201 activates integrated stress response pathway that is associated with induction of Damage Inducible Transcript 3 (DDIT3, also known as C/EBP homologous protein or CHOP). We questioned whether the ONC201/CHOP crosstalk is regulated by diverse signaling pathways in non-metastatic versus metastatic cancer cell lines. Therefore, the Dukes' type B colorectal adenocarcinoma non-metastatic (SW480) and metastatic (LS-174T) cell lines were treated with ONC201. Cell proliferation and apoptosis were evaluated by MTT assay, flow cytometry analysis, gene expression was assessed by Affymetrix microarray, and key regulatory proteins were validated by Western blot assays. Unlike LS-174T cells, SW480 cells were resistant to ONC201 treatment. Gene ontology pathway enrichment analysis of differentially expressed genes revealed substantial differences between LS-174T and SW480 responsiveness to ONC201 treatment. In both cell lines, CHOP expression was upregulated in response to ONC201 treatment, however, its upstream regulatory mechanisms were not identical. Although, PERK, ATF6 and IRE1 ER-stress pathways were found to upregulated $\mathrm{CHOP}$ in both cell types, the BAK/BAX pathway was a notable regulator of CHOP in the metastatic LS-174T cells alone. In addition, CHOP RNA splicing profiles were varied between the two cell lines, which was further modified in response to ONC201 treatments. In conclusion, we delineated the signaling mechanisms regulating the expression of CHOP in non-metastatic versus metastatic colorectal cells in response to ONC201 treatment. The observed differences were related to cellular plasticity and metabolic reprogramming.

Keywords: ONC201, colorectal cancer cells, DDIT3, CHOP, BAK/BAX pathway 


\section{Introduction}

Imipridones are a class of small molecules with anti-cancer properties. The founding member TRAIL-inducing compound 10 (TIC10), also known as ONC201, was shown to exhibit attractive physical and biochemical characteristics with selectivity toward a broad range of tumor cells but not normal cells (best reviewed in [1]. Currently, ONC201 is under evaluation in advanced clinical trials for a number of solid tumor malignancies including gliomas, recurrent or metastatic endometrial carcinoma, recurrent ovarian and peritoneal caners, refractory metastatic breast cancer, relapsed non-Hodgkin's lymphoma, and advanced neuroendocrine tumors [2]. A phase I clinical study confirmed the compound is body-tolerated, provided evidence of functionality after oral administration, revealed the desired micromolar plasma concentration for advanced cancer patients and showed enhanced immune response in patients [3].

Using human colorectal cancer cell line, ONC201 was identified while screening small molecules that induces the tumor necrosis factor (TNF)-related apoptosis-inducing ligand (TRAIL) gene expression [4]. TRAIL is an attractive anti-proliferative agent because of its ability to induce apoptosis mainly in tumor cells by activating the death receptors 4 and 5 (DR4 and DR5), with minor toxicity against normal cells [5, 6]. In solid tumor cells, ONC201 activates the Activating Transcription Factor 4 (ATF4), which is a hallmark of the integrated stress response [7]. The latter causes a dual alpha serine/threonine-protein kinase/ extracellular signal-regulated kinase (Akt/ERK) inactivation and subsequent activation of the transcription factor Forkhead box O3a (FOXO3a) which upregulates TRAIL gene expression [4]. In hematologic malignancies, ONC201 inhibits the Akt/inhibitor of apoptosis protein (IAP) pathway, downregulates the anti-apoptotic proteins Bcl-2 and $\mathrm{Bcl}-\mathrm{xl}$ and upregulates the pro-apoptotic protein Bim, which overcomes chemotherapy resistance mechanism [8, 9]. In addition, Ishizawa et al. reported that ONC201 inhibits the mammalian target of rapamycin complex 1 (mTORC1) signaling in hematological melanomas, likely through ATF4-mediated induction of the mTORC1 inhibitor DDIT4 (DNA Damage Inducible Transcript 4) [10]. In line with previous studies, ONC201 induces ATF4 in cutaneous T-cell lymphomas, which inactivates AKT as well as JAK/STAT and NF-kB pathways [11]. Independent from TRAIL mediated pro-apoptotic activity, ONC201 is reported to decrease cyclin D1 and retinoblastoma protein $(\mathrm{pRb})$ expressions, and causes cell arrest at G1 phase of the cell cycle [12]. Recently, studies have indicated a novel mechanism of action for ONC201 targeting the mitochondria [13, 14]. Breast cancer cell lines treatment with ONC201 causes mitochondria structural damage, functional impairment and gene suppression [14]. Taking together, these studies indicate that ONC201 has a broad spectrum of activity inducing several anti-proliferative signaling pathways depending on the cellular context and environment.

ONC201 exerts synergistic activity in combination with: (i) cytarabine or 5-azacytidine in AML cells [8]; (ii) Bcl-2 antagonist ABT199 in MCL-1 cells [10]; and (iii) 5-fluorouracil, 
irinotecan, oxaliplatin or RTK inhibitor crizotinib in the pancreatic cancer cell lines PANC-1 and HPAF-II [15]. Interestingly, the efficacy of ONC201 includes targeting the cancer stem cells which prime tumor initiation, relapse and metastasis. ONC201 is reported to downregulate genes associated with Wnt signaling and self-renewal in cancer stem cells from colorectal and prostate cancer and glioblastoma [16].

ONC201 treatment causes cell death by upregulating genes and proteins involved in the endoplasmic reticulum (ER) stress or integrated stress response (ISR)-related genes. The only reported ISR-ONC201 responsive factors are ATF4 and C/EBP homologous protein (CHOP) which trigger the phosphorylation and activation of the eukaryotic translation initiation factor eIF $2 \alpha[7,10]$.

The trans-activator CHOP belongs to the family of CCAAT/enhancer binding proteins (C/EBPs) and is implicated in various stress response pathways, such as ER stress [17], redox stress [18], and nutrient deprivation stress [19, 20]. CHOP plays key functional roles in apoptosis and autophagy [21, 22], as well as inhibition of adipocyte differentiation [23]. CHOP expression is regulated by basic-leucine zipper (bZIP) class transcription factors and deletion mutant analysis shows that the bZIP domain plays a critical role in CHOP-induced apoptosis [24]. The 5' flanking sequence of $C H O P$ promoter contains overlapping cis-acting CAAT enhancer binding-activating transcription factor (ATF) and cyclic AMP response element (CRE) DNA binding elements that bind to different complexes containing C/EBP $\beta$, ATF2, ATF3, and ATF4 in various cell types [25]. CHOP is ubiquitously expressed at very low levels; however, pathological conditions inducing overwhelming ER stress upregulate $\mathrm{CHOP}$ expression, resulting in apoptosis mainly regulated by upstream factors such as protein kinase RNA-like endoplasmic reticulum kinase (PERK), ATF6, and inositol requiring protein 1 (IRE1) [26]. Notably, a recent study published in bioRxiv shows that treatment of metastatic prostate cancer cell line (PC3) with ONC201 induces the expression of ATF4, ATF6 and IRE1-XBP1 signaling, upstream of CHOP (oi.org/10.1101/710400). Thus, ONC201 mechanism of action in inducing ISR proteins in cancer cells is yet to be determined.

Here, we aimed to identify the differentially expressed genes and associated signaling pathways allied with CHOP expression in colorectal cancer cell lines treated with ONC201. In this study, we used SW480 and LS-174T as cell models for primary and metastatic colorectal cancer cells, respectively. In response to ONC201 treatment, CHOP expression is upregulated in both cell lines, however, a complex process of $\mathrm{CHOP}$ regulation was observed in the metastatic cell line. Furthermore, CHOP post-transcriptional regulation by alternatively spliced isoforms was significantly altered in response to ONC201 treatment. 


\section{Results}

\subsection{ONC201 induces apoptosis in the human colorectal cancer cell lines}

Previous studies have shown that ONC201 has an anti-metastatic effect [35]. To determine if the metastatic (LS-174T) and non-metastatic (SW480) Dukes' type B colorectal adenocarcinoma cell lines are responsive to ONC201 treatment, we treated these cells with increasing concentration of ONC201 or with vehicle as control. As observed in Figure 1A, primary cell line SW480 was resistant to ONC201 toxicity and its proliferation rate was relatively sustained, independent of the drug concentration. On the other hand, metastatic LS-174T cell proliferation/viability was gradually reduced in response to the increasing concentrations of ONC201. These data suggest that ONC201 has a dose-dependent growth inhibitory effect on metastatic and only a moderate growth-inhibitory effect was observed on non-metastatic cell line.

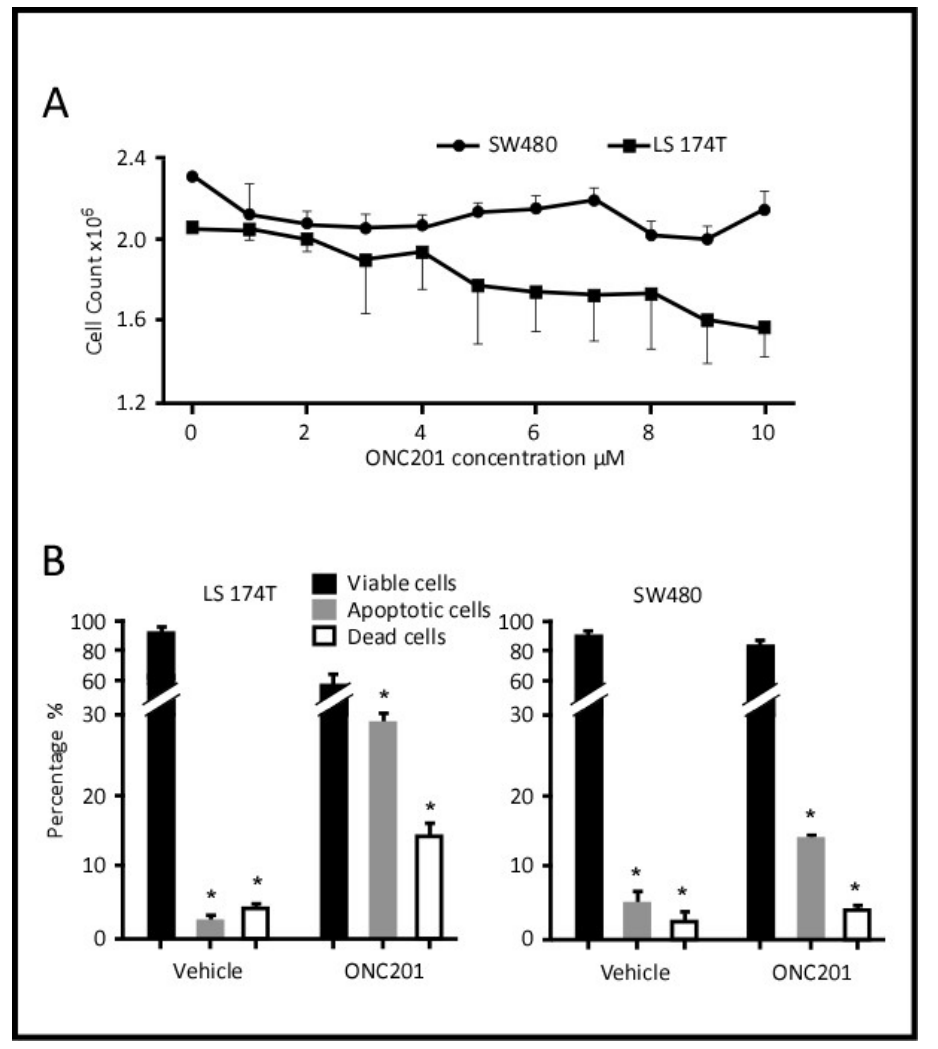

Figure 1: Response of Dukes' type B colorectal adenocarcinoma cell lines to ONC201 treatments. A. Dose-dependent response of metastatic LS-174T and non-metastatic SW480 cells to ONC201 treatment. Cells were plated in a 96-well plates, cell viability was assayed by MTT and detected using spectrophotometer. B. Flow cytometric analysis of LS-174T and SW480 cells treated with or without ONC201. Apoptotic cells were detected using annexin $\mathrm{V}$ kit, values are expressed as mean $\pm S D$. Significant values were set as ${ }^{*} \mathrm{P}$ $<0.01(\mathrm{n}=3)$.

In order to gain a better understanding of the cytotoxic effect of ONC201 on these cell lines, we used flow cytometry to evaluate cell proliferation stage in response to ONC201 treatment. As observed in Figure 1B, flow cytometric analyses were aligned with the cytotoxicity test results. Unlike non-metastatic SW480 cells showing $81 \% \pm 1.5 \%$ survival rate, the metastatic LS-174T cell viability was significantly less i.e. $57.3 \% \pm 1.8 \%$. Relative to vehicle-treated control, LS-174T cells treated with ONC201 showed a significant, 10-fold increase in apoptotic cells and 3.3-fold increase in cell death. Whereas, non-metastatic SW480 cells were more resistant to the drug treatment and the ratios of apoptotic and dead cells were only 2-2.5-fold relative to vehicle-treated control (Figure 1B). 


\subsection{Pathway enrichment of differential Microarray-based gene expression}

The studied colorectal cancer cell lines exhibited a differential response to ONC201 treatment, suggesting a unique mechanism of action which may be related to the metastatic transformation of LS-174T cells. Understanding these mechanisms may provide a new insight into the effectiveness of ONC201 treatment. Toward this end, we performed microarray transcriptome profiling on RNA samples from LS-174T and SW480 cells treated with or without ONC201 and utilized Affymetrix expression console software for data analysis, using a fold-change difference of $\geq 2$ and a P-value $<0.01$ to determine the differently-expressed genes between ONC201-treated cells versus vehicle-treated cells. This critical differentially expressed transcript filter is shown in volcano blots (Figure 2 A and B). In total, we detected 1188 upregulated gene transcripts and 1572 downregulated gene transcripts in metastatic LS-174T cells treated with ONC201 relative to vehicle-treated cells (Figure 2A). In comparison, reduced numbers of differentially regulated transcripts were observed in non-metastatic SW480 cells post ONC201 treatment, as only 519 and 379 gene transcripts were upregulated and downregulated, respectively, (Figure 2B).

A. LS 174T cells vs ONC201 treated

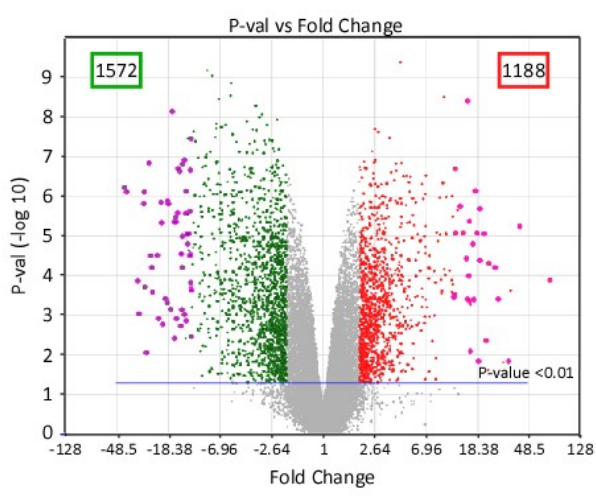

B. SW480 cells vs ONC201 treated

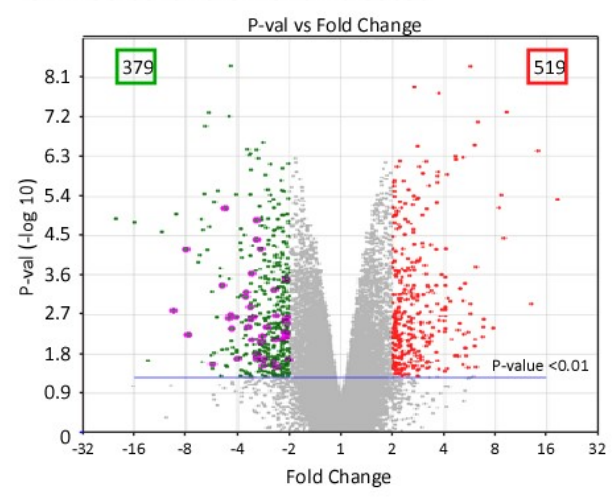

Figure 2: Affymetrix microarray analysis of LS-174T and SW480 cells treated with or without ONC201. Volcano graphs illustrating the differential gene expression in LS174T (A) and SW480 (B) cells in response to ONC201 treatment. Each dot represents one gene that had detectable expression in either cell line in response to ONC201 treatment. The horizontal line marks the threshold $(\mathrm{P}<0.01)$. The color code defines an upregulated gene as red and a downregulated gene as green, with the change $\geq$ 2-fold relative to control (vehicle-treated cells).

Next, we performed the gene ontology (GO) and Kyoto encyclopedia of genes and genomes (KEGG) pathway enrichment analysis on the differentially regulated transcripts. For both cell lines, the top signaling pathways that showed a statistically significant regulation $(p \leq 0.001)$ in response to 
ONC201 treatment are elucidated in Tables $\mathbf{1}$ and 2. Initially, these pathways were classified into major network mechanisms including oncogenesis, cell cycle, cellular metabolic pathways, DNA repair, micro RNAs, and stress; the latter was affiliated only to non-metastatic SW480 cell line treatment (Table 2). In comparison, the overall number of the regulated signaling pathways and associated genes in ONC201-treated metastatic LS-174T cells was higher than that observed for the treated primary SW480 cells.

A detailed analysis of the total gene expression profile associated with each signaling pathway revealed remarkable diversity between the metastatic and primary cancer cell lines. In drug-treated LS-174T cells, we observed a notable global downregulation of the genes associated with oncogenesis, cell cycle, and DNA repair networks. Whereas, cell homeostasis networks, such as cellular metabolic pathways and micro-RNAs showed a comparable number of upregulated or downregulated genes (Table 1). Oddly, SW480 cells treated with ONC201 showed a fewer number of regulated genes that were almost equivalently upregulated or downregulated, at least in part, for the studied networks. Notably, large numbers of stress response network genes were upregulated only in the non-metastatic SW480 cells treated with ONC201 (Table 2).

Table 1 : Top 5 gene networks differentially expressed in the Dukes' type B colorectal adenocarcinoma metastatic LS-174T cell line treated with ONC201

\begin{tabular}{|c|c|c|c|c|c|}
\hline Associated Network Functions & $\begin{array}{c}\text { Total } \\
\# \text { of } \\
\text { Genes }\end{array}$ & $\begin{array}{c}\# \text { of } \\
\text { Upregulated } \\
\text { Genes }\end{array}$ & $\begin{array}{c}\# \text { of } \\
\text { Downregulated } \\
\text { Genes }\end{array}$ & Significance & P-value \\
\hline \multicolumn{6}{|c|}{ Oncogenes } \\
\hline Retinoblastoma (RB) in Cancer & 57 & 1 & 56 & 43.82 & 0 \\
\hline Integrated Breast Cancer Pathway & 24 & 7 & 17 & 3.36 & 0.000438 \\
\hline Gastric Cancer Network 1 & 15 & 1 & 14 & 10.19 & 0 \\
\hline Gastric Cancer Network 2 & 13 & 1 & 12 & 7.31 & 0 \\
\hline $\begin{array}{l}\text { Pathways Affected in Adenoid Cystic } \\
\text { Carcinoma }\end{array}$ & 15 & 5 & 10 & 4.12 & 0.000076 \\
\hline Hepatitis C and Hepatocellular Carcinoma & 11 & 4 & 7 & 2.94 & 0.001159 \\
\hline Signaling Pathways in Glioblastoma & 13 & 7 & 6 & 2.46 & 0.003503 \\
\hline Pathways in clear cell renal cell carcinoma & 13 & 8 & 5 & 2.14 & 0.007276 \\
\hline Imatinib and Chronic Myeloid Leukemia & 6 & 3 & 3 & 2.71 & 0.001937 \\
\hline $\begin{array}{l}\text { Apoptosis-related network due to altered } \\
\text { Notch3 in ovarian cancer }\end{array}$ & 12 & 9 & 3 & 3.83 & 0.000147 \\
\hline Imatinib and Chronic Myeloid Leukemia & 6 & 3 & 3 & 2.71 & 0.001937 \\
\hline \multicolumn{6}{|c|}{ Cell Cycle } \\
\hline Cell Cycle & 35 & 0 & 35 & 15.56 & 0 \\
\hline G1 to $S$ cell cycle control & 27 & 2 & 25 & 14.02 & 0 \\
\hline Cell Cycle Checkpoints & 10 & 1 & 9 & 2.16 & 0.006869 \\
\hline HIV Life Cycle & 5 & 1 & 4 & 2.37 & 0.00431 \\
\hline
\end{tabular}


7 of 23

\begin{tabular}{|c|c|c|c|c|c|}
\hline $\begin{array}{l}\text { Regulation of sister chromatid separation at the } \\
\text { metaphase-anaphase transition }\end{array}$ & 9 & 0 & 9 & 7.07 & 0 \\
\hline Circadian rhythm related genes & 24 & 12 & 12 & 2.18 & 0.006581 \\
\hline Metapathway biotransformation Phase I and II & 24 & 14 & 10 & 2.73 & 0.001841 \\
\hline Mitotic Metaphase and Anaphase & 5 & 0 & 5 & 2.65 & 0.002233 \\
\hline \multicolumn{6}{|c|}{ Cellular Metabolic pathways } \\
\hline Pyrimidine metabolism & 25 & 2 & 23 & 8.35 & 0 \\
\hline PI3K-Akt Signaling Pathway & 36 & 22 & 14 & 2.24 & 0.005776 \\
\hline Amino Acid metabolism & 20 & 8 & 12 & 5.54 & 0.000003 \\
\hline Cholesterol Biosynthesis & 11 & 0 & 11 & 9.94 & 0 \\
\hline Cholesterol biosynthesis & 11 & 0 & 11 & 6.49 & 0 \\
\hline PPAR signaling pathway & 13 & 4 & 9 & 3.33 & 0.00047 \\
\hline Vitamin D Receptor Pathway & 22 & 14 & 8 & 2.13 & 0.007471 \\
\hline Adipogenesis & 19 & 12 & 7 & 2.93 & 0.001179 \\
\hline Nucleotide Metabolism & 8 & 1 & 7 & 4.84 & 0.000014 \\
\hline Androgen receptor signaling pathway & 13 & 7 & 6 & 2.1 & 0.007981 \\
\hline One Carbon Metabolism & 9 & 4 & 5 & 4.27 & 0.000053 \\
\hline Fatty Acid Biosynthesis & 6 & 1 & 5 & 2.6 & 0.002515 \\
\hline Exercise-induced Circadian Regulation & 10 & 6 & 4 & 2.98 & 0.001038 \\
\hline Copper homeostasis & 10 & 6 & 4 & 2.52 & 0.003042 \\
\hline GPCR ligand binding & 7 & 3 & 4 & 11.17 & 0 \\
\hline $\begin{array}{c}\text { SREBF and miR33 in cholesterol and lipid } \\
\text { homeostasis }\end{array}$ & 6 & 2 & 4 & 3.1 & 0.000787 \\
\hline $\begin{array}{c}\text { TCA Cycle and Deficiency of Pyruvate } \\
\text { Dehydrogenase complex (PDHc) }\end{array}$ & 5 & 1 & 4 & 2.52 & 0.00302 \\
\hline \multicolumn{6}{|c|}{ DNA repair } \\
\hline Histone Modifications & 19 & 0 & 19 & 7.37 & 0 \\
\hline DNA Damage Response & 20 & 4 & 16 & 8.03 & 0 \\
\hline $\begin{array}{c}\text { DNA IR-Double Strand Breaks (DSBs) and } \\
\text { cellular response via ATM }\end{array}$ & 12 & 0 & 12 & 3.44 & 0.000361 \\
\hline ATM Signaling Pathway & 11 & 1 & 10 & 4.28 & 0.000053 \\
\hline Mismatch repair & 5 & 0 & 5 & 3.89 & 0.000129 \\
\hline Homologous recombination & 6 & 1 & 5 & 4.01 & 0.000097 \\
\hline \multicolumn{6}{|c|}{ Micro RNAs } \\
\hline miR-targeted genes in lymphocytes - TarBase & 62 & 23 & 39 & 5.47 & 0.000003 \\
\hline miR-targeted genes in muscle cell - TarBase & 53 & 23 & 30 & 5.17 & 0.000007 \\
\hline miR-targeted genes in epithelium - TarBase & 46 & 20 & 26 & 4.91 & 0.000012 \\
\hline miRNA Regulation of DNA Damage Response & 21 & 4 & 17 & 5.83 & 0.000001 \\
\hline
\end{tabular}


Table 2 Top 6 gene networks differentially expressed in the Dukes' type B colorectal adenocarcinoma primary SW480 cell line treated with ONC201

\begin{tabular}{|c|c|c|c|c|c|}
\hline Associated Network Functions & $\begin{array}{c}\text { Total \# } \\
\text { of } \\
\text { Genes }\end{array}$ & $\begin{array}{c}\# \text { of } \\
\text { Upregulated } \\
\text { Genes }\end{array}$ & $\begin{array}{c}\text { \# of } \\
\text { Downregulated } \\
\text { Genes }\end{array}$ & Significance & P-value \\
\hline \multicolumn{6}{|c|}{ Oncogenes } \\
\hline Oncostatin M Signaling Pathway & 6 & 3 & 3 & 2.53 & 0.002919 \\
\hline Liver steatosis AOP & 8 & 5 & 3 & 2.74 & 0.001828 \\
\hline $\begin{array}{l}\text { TCA Cycle Nutrient Utilization and } \\
\text { Invasiveness of Ovarian Cancer }\end{array}$ & 2 & 2 & 0 & 2.36 & 0.00441 \\
\hline \multicolumn{6}{|c|}{ Cell Cycle } \\
\hline Cell Cycle & 7 & 0 & 7 & 2.14 & 0.007198 \\
\hline $\begin{array}{l}\text { Hair Follicle Development: Induction (Part } \\
\qquad 1 \text { of } 3)\end{array}$ & 5 & 1 & 4 & 2.26 & 0.005514 \\
\hline Nuclear Receptors Meta-Pathway & 17 & 6 & 11 & 3.18 & 0.00066 \\
\hline $\begin{array}{l}\text { Regulation of sister chromatid separation at } \\
\text { the metaphase-anaphase transition }\end{array}$ & 3 & 0 & 3 & 2.43 & 0.003697 \\
\hline \multicolumn{6}{|c|}{ Cellular Metabolic pathways } \\
\hline Cholesterol Biosynthesis & 5 & 0 & 5 & 4.95 & 0.000011 \\
\hline Cholesterol biosynthesis & 6 & 0 & 6 & 4.82 & 0.000015 \\
\hline Benzo(a)pyrene metabolism & 4 & 4 & 0 & 4.61 & 0.000024 \\
\hline $\begin{array}{l}\text { Sterol Regulatory Element-Binding Proteins } \\
\qquad(\text { SREBP) signaling }\end{array}$ & 9 & 3 & 6 & 4.58 & 0.000026 \\
\hline $\begin{array}{c}\text { Transcriptional cascade regulating } \\
\text { adipogenesis }\end{array}$ & 4 & 4 & 0 & 3.89 & 0.000129 \\
\hline Mevalonate pathway & 3 & 0 & 3 & 3.49 & 0.000323 \\
\hline $\begin{array}{l}\text { SREBF and miR33 in cholesterol and lipid } \\
\text { homeostasis }\end{array}$ & 4 & 0 & 4 & 3.3 & 0.000507 \\
\hline Amino Acid metabolism & 8 & 7 & 1 & 3.02 & 0.000951 \\
\hline Folate Metabolism & 6 & 2 & 4 & 2.5 & 0.003149 \\
\hline Vitamin B12 Metabolism & 5 & 1 & 4 & 2.29 & 0.005081 \\
\hline Gap junction trafficking and regulation & 4 & 1 & 3 & 2.06 & 0.008717 \\
\hline \multicolumn{6}{|c|}{ DNA repair } \\
\hline Histone Modifications & 20 & 0 & 20 & 17.15 & 0 \\
\hline \multicolumn{6}{|c|}{ Micro RNAs } \\
\hline miR-targeted genes in epithelium - TarBase & 26 & 13 & 13 & 7.22 & 0 \\
\hline miR-targeted genes in muscle cell - TarBase & 25 & 11 & 14 & 5.28 & 0.000005 \\
\hline $\begin{array}{c}\text { miR-targeted genes in lymphocytes - } \\
\text { TarBase }\end{array}$ & 28 & 13 & 15 & 5.22 & 0.000006 \\
\hline miR-targeted genes in leukocytes - TarBase & 13 & 5 & 8 & 4.24 & 0.000058 \\
\hline
\end{tabular}




\begin{tabular}{|c|c|c|c|c|c|}
\hline ATF4 activates genes & 4 & 3 & 1 & 2.33 & 0.004698 \\
\hline $\begin{array}{l}\text { Photodynamic therapy-induced unfolded } \\
\text { protein response }\end{array}$ & 10 & 9 & 1 & 9.93 & 0 \\
\hline Exercise-induced Circadian Regulation & 6 & 5 & 1 & 3.26 & 0.000545 \\
\hline White fat cell differentiation & 4 & 3 & 1 & 2.33 & 0.004698 \\
\hline VEGFA-VEGFR2 Signaling Pathway & 12 & 8 & 4 & 2.24 & 0.005807 \\
\hline EGF/EGFR Signaling Pathway & 11 & 6 & 5 & 3.1 & 0.000787 \\
\hline
\end{tabular}

In response to ONC201 treatment, observed differences between two cells lines suggest the implication of differentially regulated mechanisms in these cell types. Accordingly, we performed a comparative meta-analysis in the differentially regulated singling pathways in both cell lines. Figure 3A shows the cellular metabolic pathways that were primarily and significantly regulated in both cell lines in response to ONC201 treatment, with $p$-values ranging between $1 \times 10^{-24}$ and $3.291 \times 10^{-7}$ for LS-174T and SW480 cells, respectively. Signaling pathways associated with metabolic regulation and cell cycle were among the top ten significantly regulated pathways in both cell lines in response to the drug treatment (Figure 3A). Remarkably, non-metastatic SW480 cells illustrated a smaller number of signaling pathways that were significantly regulated post ONC201 treatment as compared to metastatic LS-174T cells (Figure 3A).

Then, we performed comparative analysis to decipher genes associated with the differentially regulated pathways and ranked them in accordance with their $p$-value. The top 15 genes that were significantly upregulated in either cell line in response to ONC201 are listed in Figure 3B. We noticed that FAM129A, DDIT3 and ASNS gene transcripts were upregulated in both cell lines with $\mathrm{p}$ $\leq$ 0.001. FAM129A (known as NIBAN1, Niban Apoptosis Regulator 1) encodes a highly expressed protein in cancer. Since the cell lines are carcinogenic in nature, such a transcript is expected to be detected in our microarray data. DDIT3/CHOP is a transcription factor and a member of the CCAAT/enhancer-binding protein (C/EBP) family. ASNS (Asparagine Synthetase) is involved in asparagine synthesis and facilitates progression through G1 phase of the cell cycle (Figure 3B). 
A. Alignment of ONC201-mediated Signaling Pathways changes in LS174T and SW480

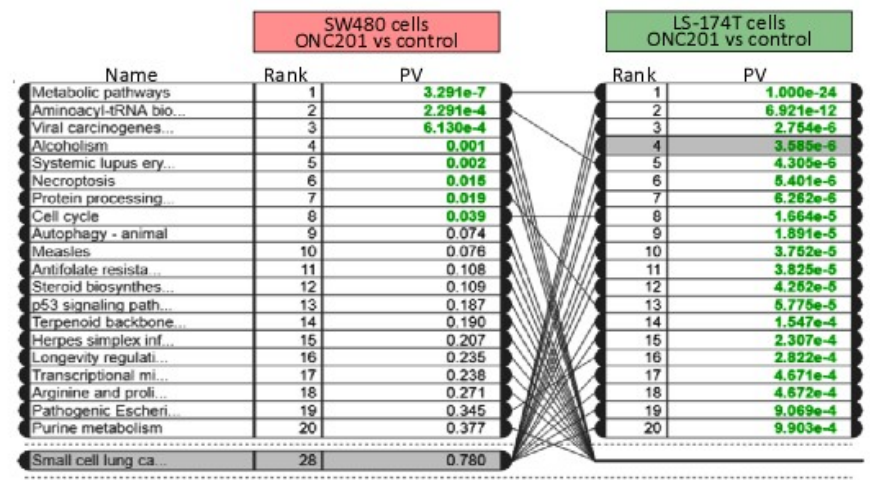

B. Alignment of ONC201- mediated Gene Expression changes in LS-174T and SW480

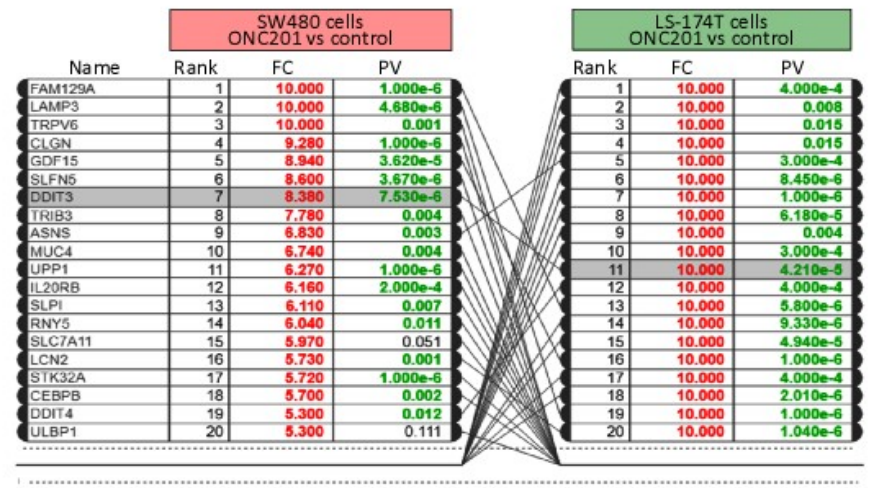

Figure 3: Meta-analysis Alignment of the differentially expressed common pathways and genes. A. Top ranked pathways that are differentially regulated in metastatic LS-174T cells and non-metastatic SW480 cells in response to ONC201 treatment are shown, with significantly low P-values in green color. B. Top ranked transcripts differentially regulated in LS-174T and SW480 cells in response to ONC201 treatment are shown, with significantly low P-values in green and fold expression in red color.

Our initial results indicated that ONC201 induces apoptosis differentially in both cell lines and since $\mathrm{CHOP}$ is a critical regulatory factor for this pathway, therefore, we focused our study on the regulatory mechanisms associated with this gene. Mapping the differentially-expressed gene transcripts detected in microarray data onto the map of ER protein processing pathway [36, 37] exposed similar, but not identical, regulatory mechanisms upstream of $\mathrm{CHOP}$ for each treated cell line (Figure 4,). In metastatic LS-174T cells, ONC201 treatment induced the upregulation of CHOP transcripts through the upregulation of inositol-requiring protein 1 (IRE1), activating transcription factor 6 (ATF6), protein kinase R-like endoplasmic reticulum kinase (PERK), and BAK/BAX signaling networks (Figure 4A). On the other hand, these regulatory mechanisms were also observed in ONC201-treated SW480 cells, but not the BAK/BAX pathway expression (Figure 4B). In 
addition, transcripts of the down-stream anti-apoptotic BCL2 were significantly downregulated in metastatic LS-174T cells, relative to those in non-metastatic SW480 cells, post ONC201 treatments.

A. Pathway analysis of genes differentially expressed in LS 174T cells treated with ONC201

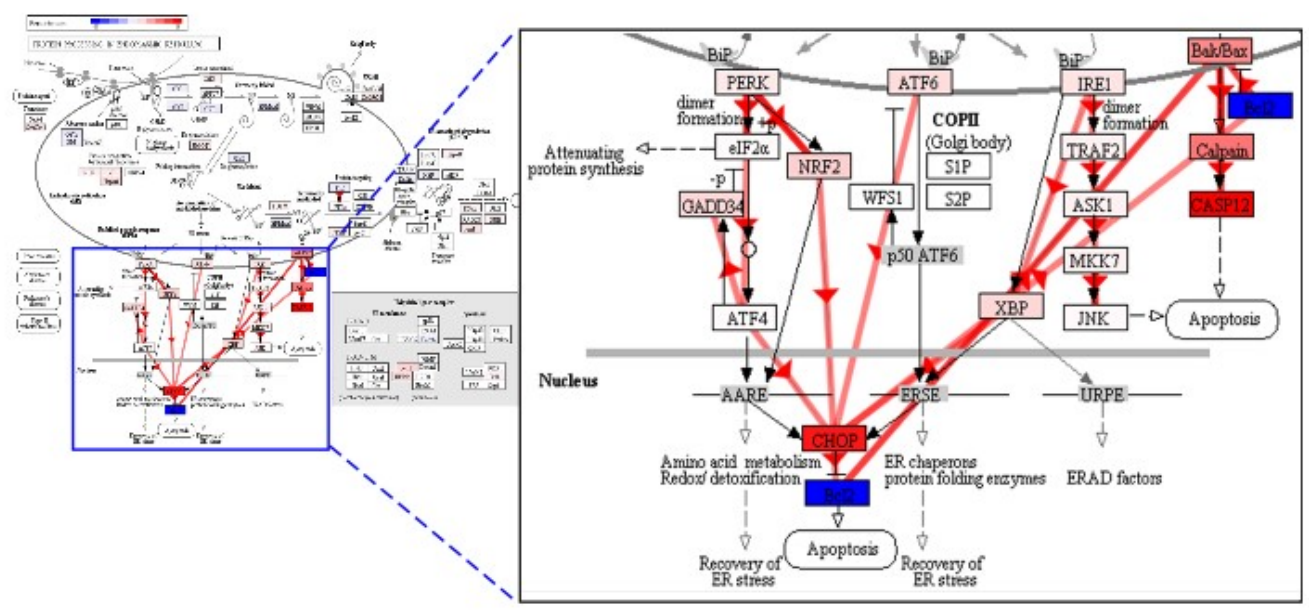

B. Pathway analysis of genes differentially expressed in SW480 cells treated with ONC201

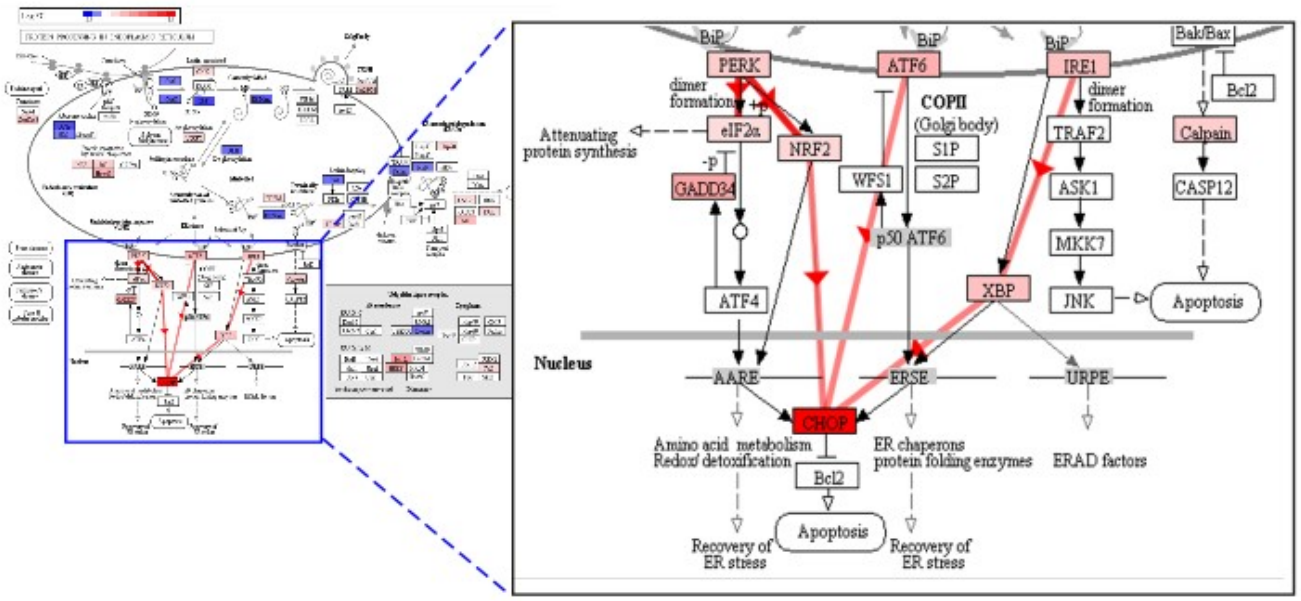

Figure 4: Schematic showing CHOP regulation in response to ONC201 treatment. A. Pathway analysis of genes differentially expressed in metastatic colorectal cancer LS174T cells treated with ONC201. B. Pathway analysis of genes differentially expressed in non-metastatic colorectal cancer SW480 cells treated with ONC201.

\subsection{ONC201 treatment elicits apoptosis in LS-174T and SW480 human colorectal cancer cells}

The observed microarray gene expression data were validated by Western blot analysis for selected proteins associated with the upregulation of CHOP. Indeed, treatment with ONC201 resulted in a significant concentration-dependent increase in CHOP protein expressions in both LS-174T cells (Figure 5) and SW480 cells (Figure 6). The upstream CHOP regulatory proteins associated with different ER signaling pathways were also studied and have shown similar pattern of expression as 
observed in the microarray study. In this regard, ATF6 protein expression increased in response to ONC201 treatments in both cell lines, and it was statistically significant when non-metastatic SW480 cells were treated with $20 \mu \mathrm{M}$ of ONC201. Alternatively, the BAX proteins were significantly increased in metastatic LS-174T cells post ONC201 treatments, but not in non-metastatic SW480 cells (Figures 5 and 6). PERK regulated proteins (eIF2 $\alpha$, GADD34, and ATF4) were differentially expressed in response to ONC201 treatment of these cell lines. EIF2 $\alpha$ protein expression was significantly increased in non-metastatic SW480 cells (Figure 6) while it was significantly reduced in metastatic LS-174T cells at $20 \mu \mathrm{M}$ of ONC201 (Figures 5). Alternatively, low ONC201 concentration treatments were associated with a slight reduction of ATF4 expression in both cell lines, however, at high ONC201 concentration $(20 \mu \mathrm{M})$, a significant reduction was observed in metastatic LS-174T cells only (Figure 5). On the other hand, GADD34 expression was significantly augmented in both cell lines in response to ONC201 treatment (Figures 5 and 6). Microarray analysis indicated a significant reduction in BCL2 transcripts, particularly in ONC201 responsive metastatic LS-174T cells. This was further confirmed at protein level whereby BCL2 protein was significantly downregulated post ONC201 treatments (Figure 5), Alternatively, BCL2 protein expression was sustained in the presence or absence of ONC201 treatments in non-metastatic SW480 cells (Figure 6). Together, microarrays analysis data and protein expression levels of the studied signaling network markers were found in alignment after ONC201 treatments. 

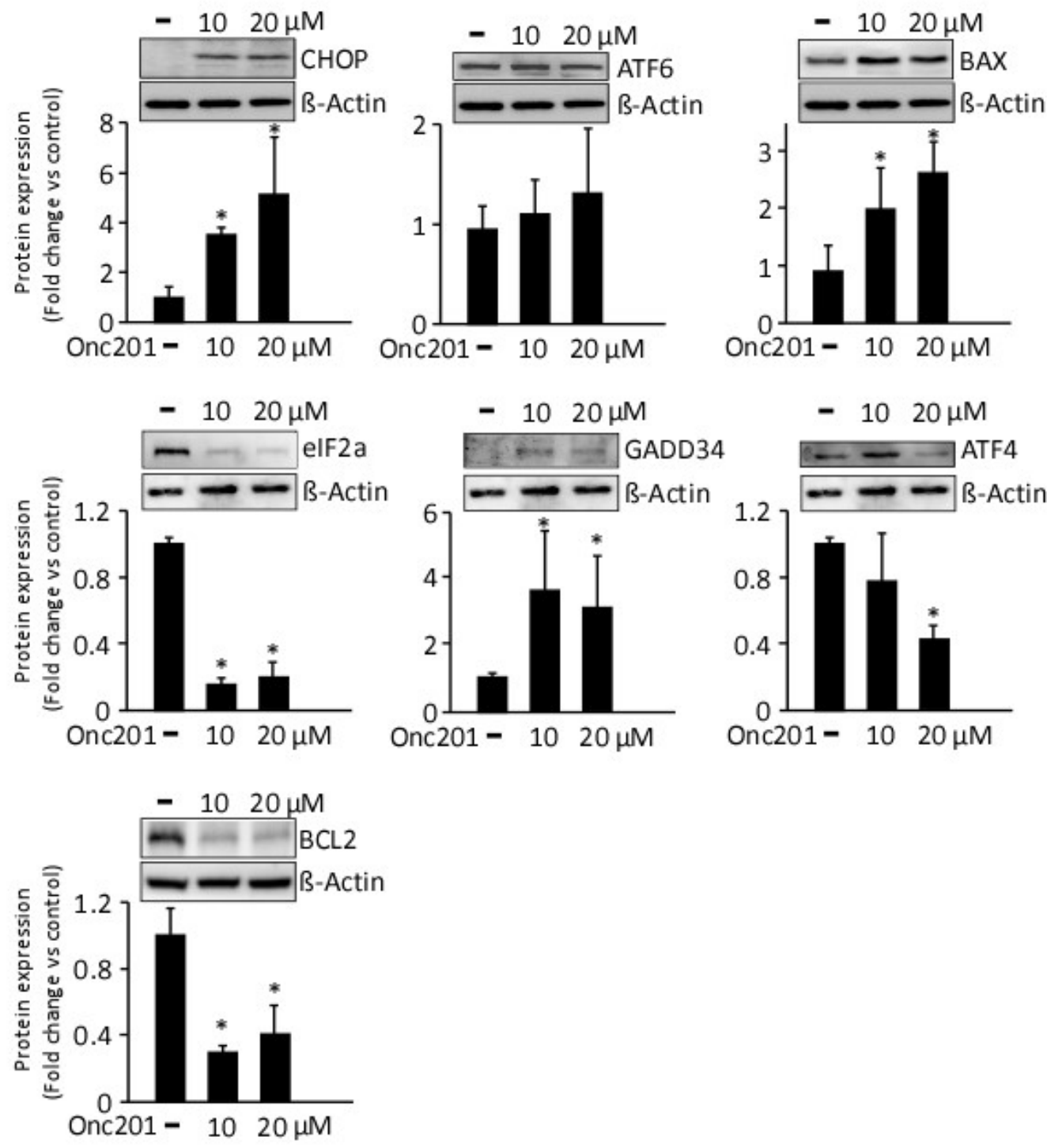

Figure 5: Western bolt analysis of metastatic LS-174T cells treated with or without ONC201, at varying concentrations. Differential expression patterns of different proteins that are involved in CHOP regulation and ER stress are shown. Densitometric analysis of the representative Western blots is shown (mean $\pm \mathrm{SD})$, with respect to housekeeping gene $\beta$-actin in different treatment groups $(n=3)$. Significant values were set as ${ }^{*} \mathrm{P}<0.01$. 

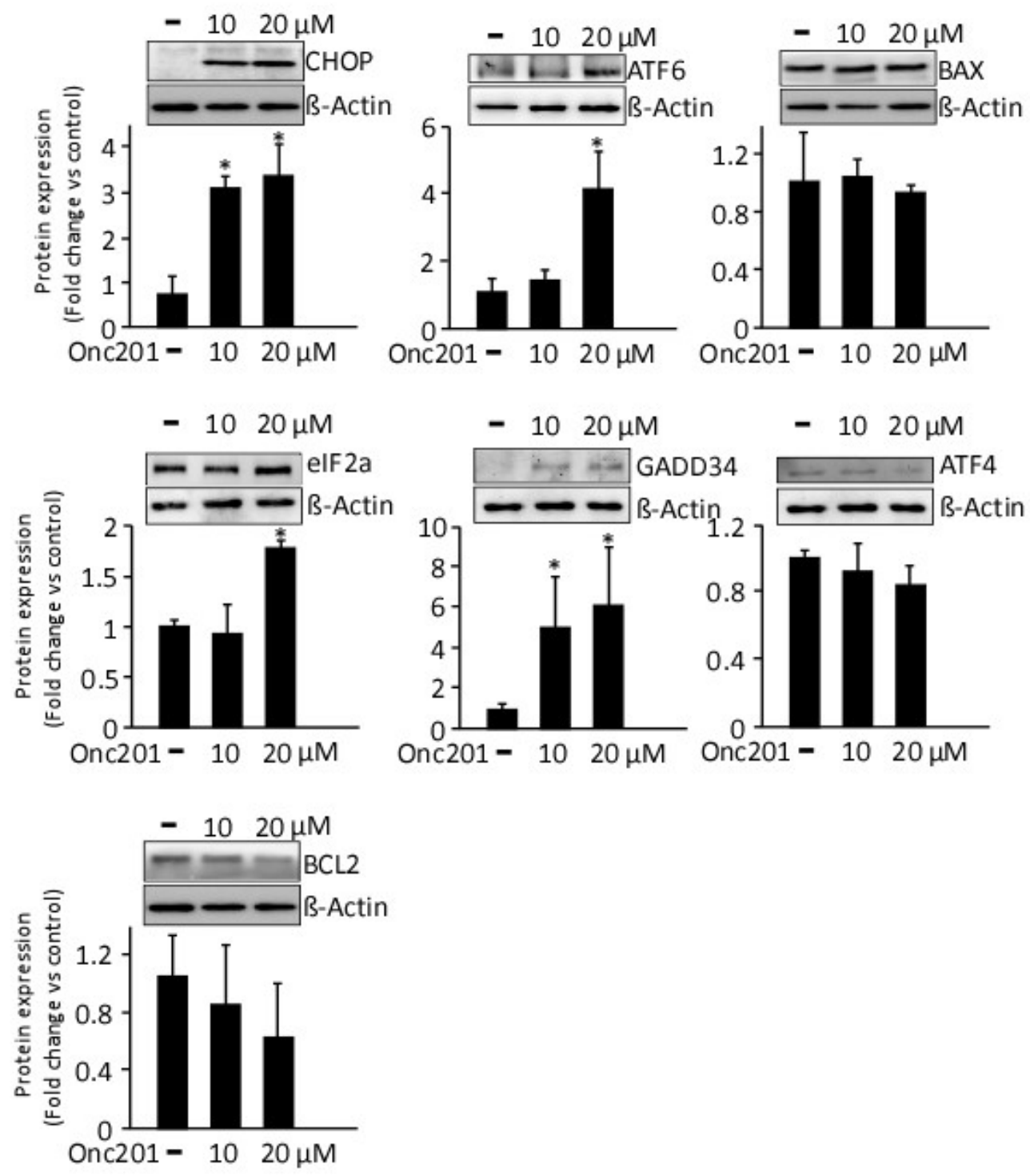

Figure 6: Western blot analysis of non-metastatic SW480 cells treated with or without ONC201, at varying concentrations. Differential expression patterns of different proteins involved in CHOP regulation and ER stress are shown. Densitometric analysis of the representative Western blots is shown (mean $\pm S D$ ), with respect to housekeeping gene $\beta$-actin in different treatment groups $(n=3)$. Significant values were set as ${ }^{*} \mathrm{P}<0.01$ 


\subsection{Alternative Splicing CHOP mRNA}

Defects in the RNA alternative splicing are a hallmark of cancerous cells. Many RNA splicing regulators are studied as tumor suppressors or being associated with drug resistance [38-40]. Exon splicing analysis of CHOP showed significant variation derived from metastatic LS-174T cells treated with vehicle versus ONC201, with a significantly reduced (up to 4.65-fold) splicing index signal levels in exon 2 in samples treated with the drug (Figure 7A). In order to further confirm these data experimentally, we performed quantitative RT-PCR and fractionated the products on a bioanalyzer. As observed in Figure 7B, a differential splicing pattern of CHOP mRNA has been observed in metastatic LS-174T and non-metastatic SW480 cells, which is further modified in response to ONC201 treatment. This is an additional indication of the multifaced mechanisms of action of the ONC201 as an anti-cancer drug.

A

Symbol: CHOP, chromosome 12: 57,970,731 - 57,914,300

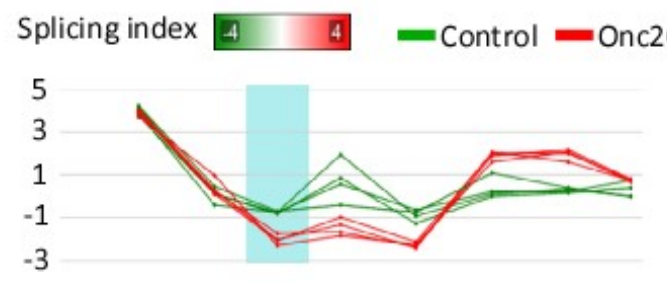

LS 174T splicing Index (Gene-level Signal: 8.38)

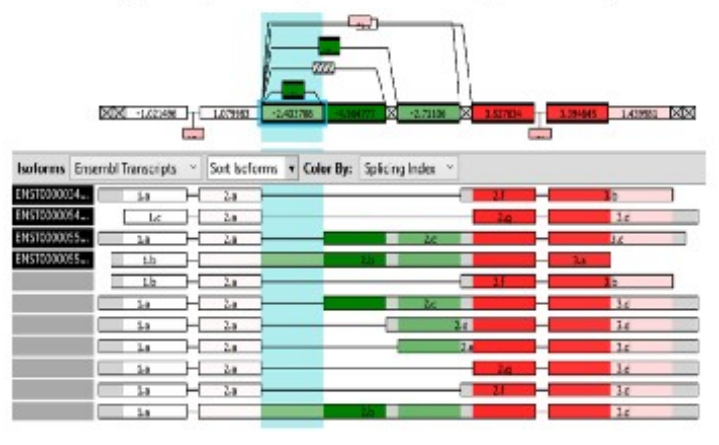

B

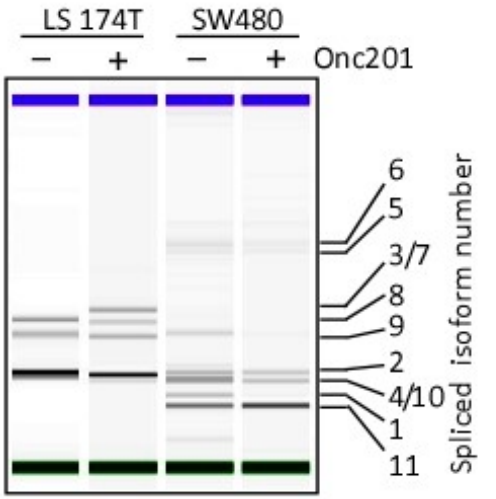

Figure 7: Alternative spliced variants of CHOP. A. A computational analysis detecting CHOP differential alternative splicing events obtained from the microarray study of SW480 treated with and without ONC201. B. Bioanalyzer electrophoresis after quantitative RT-PCR analysis of LS-174T and SW480 cells treated with and without ONC201. CHOP was amplified using specific primers flanking the exons 1 and 4. RT-PCR products were fractionated on Bioanalyzer microgel. Alternative spliced isoforms were mapped according to their fragment size. 
16 of 23

\section{Discussion}

Using non-metastatic (SW480) and metastatic (LS-174T) colorectal cancer cell lines, we identified the differential mechanisms of CHOP regulation in response to ONC201 treatment which, in turn, downregulates BCL2 and induces apoptosis. It has been documented that ONC201 treatment interrupts the ER homeostasis and induces expression of the three ER stress response signaling networks PERK, ATF6 and IRE1, known to trigger the unfolded protein response (UPR) signaling [41, 42]. Here, we report a prospective role for the ER stress Bak/Bax network in targeting CHOP regulation in the metastatic colorectal cell line LS-174T after treatment with ONC201. Additionally, the microarray data indicated a prospective crosstalk between IRE1 and Bak/Bax signaling pathways in LS-174T cells. Furthermore, the complexity of the CHOP regulatory mechanism in the metastatic cell line and the subsequent downregulation of BCL2 may explain the observed proliferation arrest and high apoptosis rates in LS-174T cells in response to ONC201 treatment as compared to that of non-metastatic cell line SW480. We observed that BCL2 was consistently downregulated in metastatic LS-174T cells. BAK/BAX autoactivation can occur, independently of the activator BH3s (BIM, BID, PUMA, and NOXA), following BCL2 downregulation [43]. It thus remains unclear whether the expression and activity of BH3s is modulated in metastatic LS-174T cells post ONC201 treatment.

Our observations are in accordance with previous studies highlighting the role of ONC201 in mediating ER stress response in breast cancer cells [12, 44] and high-grade central nervous system glioblastoma [45]. However, in these studies, the observed ER stress response was primarily due to ATF4 activation. Lev et. al. (2017) compared ONC201 sensitive HAPF-II versus resistant PANC-1 pancreatic cell lines and reported a discrepancy in the ER stress response as ONC201 mediated upregulation of the three ER-stress response signaling molecules in PANC-1 cells; while on the contrary, ATF4 was the only protein to be upregulated in HPAF-II cells, with no substantial expression of IRE1 or ATF6 proteins [15]. These data suggest that the cellular response to ONC201 treatment is cell type-dependent, but the overall mechanisms are associated with ER stress and unfolded protein response signaling.

Gene expression profiles in colorectal cancer cells revealed that ONC201 downregulates genes that are associated with energy metabolism. ONC201 reduced the gene expression of citrate carrier (SLC25A1) and fumarate hydratase (FH) that regulate the mitochondrial metabolite carrier and substrate metabolism, respectively. SLC25A1 is involved in citrate mitochondria/cytoplasm translocations for cellular energy homeostasis [46]. FH plays an important role in the Krebs cycle providing FADH and NADH to the electron transport chain for ATP production [47]. Thus, ONC201 is involved in reducing metabolic pathways that may cause energy stress in cancer cells. In a similar finding, Ishida et al. observed a decrease in the ATP levels associated with low glycolysis and oxidative phosphorylation that caused energy stress to cancer cells [48]. Furthermore, ONC201 can reduce mitochondrial respiration in breast cancer cells that may lead to energy stress (reducing ATP) and result in apoptosis [14].

Transcriptome Analysis Console software, confirmed by RT-PCR analysis, identified several spliced variants of $\mathrm{CHOP}$ that are differentially expressed in the metastatic and primary colorectal cell lines 
in response to OC201 treatments. Notably, exon 2 is the target for CHOP splicing mechanism. The function of exon 2 of $\mathrm{CHOP}$ is unclear, though the exon encodes part of the $5^{\prime}$-untranslated region, and in general, the untranslated regions are ventured to regulate the protein translation activity or mRNA expression $[49,50]$. CHOP belongs to the CCAAT/enhancer-binding protein (C/EBP) family of transcription factors and functions as a dominant-negative inhibitor by forming heterodimers with other $\mathrm{C} / \mathrm{EBP}$ members. Alternative splicing has been reported for other family members. C/EBP epsilon gene is regulated by alternative translational initiation site and splicing mechanisms [51] which generate four different isoforms with different functions [52, 53]. In addition to alternative translational initiation, the expression of four alternative C/EBP $\varepsilon$ isoforms (p32, p30, p27, and p14) was attributed to differential promoter usage and alternative splicing [54].

In summary, the efficacy of the outcome of cancer treatment is dependent on the stage of the disease. The differences between primary and metastatic cancer cells are affiliated to cellular plasticity and metabolic reprogramming [55], indicating a differential response to chemotherapy as observed in this and other studies. In this study, we delineated the signaling mechanisms and associated genes that differentially regulate $\mathrm{CHOP}$ expression in non-metastatic and metastatic colorectal adenocarcinoma cells, leading to increased expression of the core regulators, Bak and Bax, of the intrinsic pathway of apoptosis in metastatic LS-174T cells treated with ONC201.

\section{Materials and Methods}

\subsection{Cancer cell lines and tissue culture}

Dukes' type B colorectal adenocarcinoma cell lines LS-174T (ATCC $®$ CL-188 ${ }^{\mathrm{TM}}$ ) and SW480 (ATCC ${ }_{\text {) }}$ CCL-228 ${ }^{\mathrm{TM}}$ ) were purchased from the American Type Culture Collection (ATCC, Manassas, VA, USA). Cells were grown in RPMI1640 media (Invitrogen Corporation, Carlsbad, CA, USA) supplemented with $10 \%$ fetal bovine serum (FBS; Invitrogen Corporation) and $100 \mathrm{U} / \mathrm{mL}$ of penicillin-streptomycin (Gibco, Carlsbad, CA, USA) and incubation at $37^{\circ} \mathrm{C}$ in $5 \% \mathrm{CO}_{2}$ incubator. ONC201 (SML1068, Sigma Aldrich, Germany) was dissolved in dimethyl sulfoxide (DMSO, D8418, Sigma Aldrich, Germany) to a stock concentration of $10 \mathrm{mM}$ and stored at $-20^{\circ} \mathrm{C}$ in aliquots. The control (vehicle) used was $0.01 \%$ DMSO. Cells were treated with ONC201 at concentrations of $10 \mu \mathrm{M}$ and $20 \mu \mathrm{M}$ for $24 \mathrm{~h}$ before initiation of experiments while controls were treated with vehicle i.e. $0.01 \%$ DMSO.

\subsection{Preparation of Protein Extract and Western blot Analysis}

Cells were harvested and lysed using modified RIPA buffer (50 mM Tris-HCl pH 7.5, $150 \mathrm{mM}$ $\mathrm{NaCl}, 1 \%$ Triton x100, $1 \mathrm{mM}$ EDTA, $0.5 \%$ Sodium deoxycholate and $0.1 \%$ SDS). Cell lysates were quantified using the Pierce BCA Protein Assay Kit (Thermo Fisher Scientific GmbH, Driesch, Germany) and equal amounts of protein $(30 \mu \mathrm{g})$ were resolved on $8 \%-12 \%$ polyacrylamide gels and transferred to polyvinylidene fluoride membranes (EMD Millipore Corporation, Billerica, MA, USA) as described previously [27]. After blocking, membranes were blotted with the corresponding primary and horseradish peroxidase-linked secondary antibodies [28]. Primary antibodies used were: $\beta$-actin (ab8224), eIF2 $\alpha$ (ab169528), GADD34 (ab9869) and ATF4 (ab85049); purchased from Abcam, USA. CHOP (2895S), BAX D2E11 (5023T) and BCL-2 D55G8 (4223T) were from Cell 
Signaling, USA; ATF6 (ALX-804-381-C100) was purchased from ANZO, USA. Finally, immunoblots were detected by chemiluminescence using Chemidoc MP system (Bio-Rad, USA). ImageJ V1.49O software (https://imagej.nih.gov/ij/) was used to quantify the immunoblot signals as a mean of the grey/white scale of all the pixels in a band [29].

\subsection{MTT Cytotoxicity assay}

Cytotoxicity assays were performed as described previously [30]. Briefly, cells were seeded into a 96-well plate at a density of $10^{6}$ cells/well at $37^{\circ} \mathrm{C}$ in $5 \% \mathrm{CO}_{2}$ for $12 \mathrm{~h}$. Then, control wells were treated with vehicle and experimental wells with increasing concentrations (1 to $10 \mu \mathrm{M})$ of ONC201. After $48 \mathrm{~h}, 1 \mathrm{mg} / \mathrm{ml}$ of MTT (3-[4,5-dimethylthiazol-2-yl]-2,5-diphenyltetrazolium, Promega Bio Sciences LLC, San Luis Obispo, CA, USA) was added in each well. The developed Formazan crystals were subsequently dissolved in $10 \% \mathrm{SDS} / 0.04 \mathrm{eq} / \mathrm{L} \mathrm{HCl}$ solution for $1 \mathrm{~h}$ at $37^{\circ} \mathrm{C}$. Absorbance was measured at $490 \mathrm{~nm}$ using a microplate reader (DTX880; Beckman Coulter, Brea ,Ca, USA). Cell survival was expressed as the percentage of control cells [CS $=\left(\right.$ Mean $A_{\text {treated well }} /$ Mean $\left.A_{\text {control well }}\right) \mathrm{X}$ $100 \%]$.

\subsection{Fluorescence-activated cell sorting (FACS)}

To quantify apoptosis, cells were stained with annexin V kit (Abcam, Cambridge, MA, USA), according to the manufacturer's protocol. Briefly, $1 \times 10^{5}$ cells were treated with $10 \mu \mathrm{M}$ of ONC201 for $48 \mathrm{~h}$. Cells were collected after trypsinization and washed twice with PBS. Cell pellets were resuspended in $100 \mu \mathrm{L}$ of $1 \times$ annexin-binding buffer and $1 \mu \mathrm{L}$ of an annexin V-Fluorescein isothiocyanate (FITC) working solution was added to each $100 \mu \mathrm{L}$ of cell suspensions. The suspensions were incubated on ice for $10 \mathrm{~min}$ in dark. The cell suspension volume was brought to $250 \mu \mathrm{L}$ with $1 \times$ binding buffer. The stained cells were placed on a glass slide and covered with a glass coverslip. The cells were observed under a fluorescence microscope using a filter set for FITC detection. Alternatively, the stained cells were immediately analyzed by flow cytometry FACS Calibur (BD Biosciences, Becton Dickinson, Franklin Lakes, NJ USA). For each measurement, at least 20,000 cells were counted. After drug treatment, cells were collected and incubated with propidium iodide (PI) and Annexin V (Biolegend, USA) and analyzed by flow cytometry. The percentage of apoptosis was calculated by the formula: (\% apoptosis $=\%$ PI and Annexin V double positive cells with drug - \% PI and Annexin V double positive cells without drug).

\subsection{RNA extraction, Microarray assay and q-PCR assays}

Total RNA was isolated using Trizol-Chloroform method as described in [31]. The isolated RNA was quantified, and RNA integrity was assessed by microfluidic analysis using Bioanalyser 2100 (Agilent Technologies, Santa Clara, CA, United States). For microarray assay, total RNA (100 ng) from each sample (in triplicate) were reverse transcribed as per the manufacturer's protocol (GeneChip WT PLUS Reagent, Thermo Fisher Scientific). Purified cDNA was fragmented, labeled, and hybridized onto GeneChip Human Transcriptome Array 2.0 (Thermo Fisher Scientific GmbH) for $16 \mathrm{~h}$ at $45^{\circ} \mathrm{C}$ in Affymetrix GeneChip Hybridization Oven 640 at 60 revolutions per minute (rpm). Chips were then washed and stained using Affymetrix GeneChip Fluidics Station 450 (Thermo Fisher Scientific) and 
scanned with Affymetrix GeneChip Scanner 3000 7G (Thermo Fisher Scientific). CEL data files were analyzed using Affymetrix Expression Console Software provided by the manufacturer.

For the spliced isoforms analysis, cDNA was synthesized from $1 \mu \mathrm{g}$ RNA by reverse transcription using QuantiTect Reverse Transcription Kit (Qiagen Inc., Hilden, Germany) as described [32]. Reverse transcribed RNA was used as a template for CHOP spliced isoforms quantitative PCR amplification using specific primers and FastStart SYBR Green kit (Roche Applied Sciences, Penzberg, Germany). Forward (5'-TAAGGCACTGAGCGTATCATG-3') and reverse (5'-CTGGACAGTGTCCCGAAGGAGAAA-3') primers were designed using Primer Bank [33]. q-PCR products were run on Agilent 2100 Bioanalyzer system using High Sensitivity DNA electrophoresis kit as described by the manufactures (Agilent Technologies) and quantified by High Sensitivity DNA assay software.

\subsection{Statistical Analysis}

All experiments and assays were done in technical duplicates or triplicates for three biological samples. Results were compounded and statistical significance was estimated with a two-tailed Student's $t$-test assuming equal variance performed in GraphPad Prism version 8.0. Data were presented as mean \pm standard error of mean (SEM) as previously described [34].

Author Contribution: F.A-M, A.A.M and M.B. contributed to the study design. D.H., M.A.T., S.J., W.A.A., R.N., L.M., F.A-R., S.S. and R.A. were involved in data acquisition, and/or discussion, drafting and editing. A.A.M., R.A. M.B. and F.A-M were involved in data analysis and interpretation. A.A.M. and F.A-M wrote the manuscript, S.S., R.A. and M.B. revised manuscript. All authors contributed to the drafting and critical review of the manuscript and approved the final draft.

Funding: This study was funded by Kuwait Foundation for the Advancement of Sciences (KFAS) and Dasman Diabetes Institute (DDI), grant number RA-HM-2018-039.

Acknowledgments: The authors would like to thank Kuwait university and Dasman Diabetes Institute for providing the support and laboratory space.

Conflicts of Interest: The authors declare that they have no competing interests.

Ethics approval and consent to participate: Not applicable

Availability of data and materials: All data generated and analyzed during this study are included in this article. 


\section{References}

1. Allen, J.E., et al., Discovery and clinical introduction of first-in-class imipridone ONC201. Oncotarget, 2016. 7(45): p. 74380-74392.

2. Arrillaga-Romany, I., et al., A phase 2 study of the first imipridone ONC201, a selective DRD2 antagonist for oncology, administered every three weeks in recurrent glioblastoma. Oncotarget, 2017. 8(45): p. 79298-79304.

3. Stein, M.N., et al., First-in-Human Clinical Trial of Oral ONC201 in Patients with Refractory Solid Tumors. Clin Cancer Res, 2017. 23(15): p. 4163-4169.

4. Allen, J.E., et al., Dual inactivation of Akt and ERK by TIC10 signals Foxo3a nuclear translocation, TRAIL gene induction, and potent antitumor effects. Sci Transl Med, 2013. 5(171): p. 171ra17.

5. Holland, P.M., Death receptor agonist therapies for cancer, which is the right TRAIL? Cytokine Growth Factor Rev, 2014. 25(2): p. 185-93.

6. Allen, J.E., R.N. Crowder, and W.S. El-Deiry, First-In-Class Small Molecule ONC201 Induces DR5 and Cell Death in Tumor but Not Normal Cells to Provide a Wide Therapeutic Index as an Anti-Cancer Agent. PLoS One, 2015. 10(11): p. e0143082.

7. Kline, C.L., et al., ONC201 kills solid tumor cells by triggering an integrated stress response dependent on ATF4 activation by specific eIF2alpha kinases. Sci Signal, 2016. 9(415): p. ra18.

8. Prabhu, V.V., et al., Single agent and synergistic combinatorial efficacy of first-in-class small molecule imipridone ONC201 in hematological malignancies. Cell Cycle, 2018. 17(4): p. 468-478.

9. Allen, J.E., et al., Genetic and Pharmacological Screens Converge in Identifying FLIP, BCL2, and IAP Proteins as Key Regulators of Sensitivity to the TRAIL-Inducing Anticancer Agent ONC201/TIC10. Cancer Res, 2015. 75(8): p. 1668-74.

10. Ishizawa, J., et al., ATF4 induction through an atypical integrated stress response to ONC201 triggers p53-independent apoptosis in hematological malignancies. Sci Signal, 2016. 9(415): p. ra17.

11. Ni, X., et al., ONC201 selectively induces apoptosis in cutaneous T-cell lymphoma cells via activating pro-apoptotic integrated stress response and inactivating JAK/STAT and NF-kappaB pathways. Oncotarget, 2017. 8(37): p. 61761-61776.

12. Ralff, M.D., et al., ONC201 Demonstrates Antitumor Effects in Both Triple-Negative and Non-Triple-Negative Breast Cancers through TRAIL-Dependent and TRAIL-Independent Mechanisms. Mol Cancer Ther, 2017. 16(7): p. 1290-1298.

13. Graves, P.R., et al., Mitochondrial Protease ClpP is a Target for the Anticancer Compounds ONC201 and Related Analogues. ACS Chem Biol, 2019. 14(5): p. 1020-1029.

14. Greer, Y.E., et al., ONC201 kills breast cancer cells in vitro by targeting mitochondria. Oncotarget, 2018. 9(26): p. 18454-18479.

15. Lev, A., et al., Anti-pancreatic cancer activity of ONC212 involves the unfolded protein response (UPR) and is reduced by IGF1-R and GRP78/BIP. Oncotarget, 2017. 8(47): p. 81776-81793. 
16. Prabhu, V.V., et al., Cancer stem cell-related gene expression as a potential biomarker of response for first-in-class imipridone ONC201 in solid tumors. PLoS One, 2017. 12(8): p. $\mathrm{e} 0180541$.

17. Tang, Q.Q. and M.D. Lane, Role of C/EBP homologous protein (CHOP-10) in the programmed activation of CCAAT/enhancer-binding protein-beta during adipogenesis. Proc Natl Acad Sci U S A, 2000. 97(23): p. 12446-50.

18. McCullough, K.D., et al., Gadd153 sensitizes cells to endoplasmic reticulum stress by down-regulating Bcl2 and perturbing the cellular redox state. Mol Cell Biol, 2001. 21(4): p. 1249-59.

19. Averous, J., et al., Induction of CHOP expression by amino acid limitation requires both ATF4 expression and ATF2 phosphorylation. J Biol Chem, 2004. 279(7): p. 5288-97.

20. Bruhat, A., et al., Amino acid limitation induces expression of CHOP, a CCAAT/enhancer binding protein-related gene, at both transcriptional and post-transcriptional levels. J Biol Chem, 1997. 272(28): p. 17588-93.

21. Marciniak, S.J., et al., CHOP induces death by promoting protein synthesis and oxidation in the stressed endoplasmic reticulum. Genes Dev, 2004. 18(24): p. 3066-77.

22. Ogbechi, J., et al., Inhibition of Sec61-dependent translocation by mycolactone uncouples the integrated stress response from ER stress, driving cytotoxicity via translational activation of ATF4. Cell Death Dis, 2018. 9(3): p. 397.

23. Carriere, A., et al., Mitochondrial reactive oxygen species control the transcription factor CHOP-10/GADD153 and adipocyte differentiation: a mechanism for hypoxia-dependent effect. J Biol Chem, 2004. 279(39): p. 40462-9.

24. Matsumoto, M., et al., Ectopic expression of CHOP (GADD153) induces apoptosis in M1 myeloblastic leukemia cells. FEBS Lett, 1996. 395(2-3): p. 143-7.

25. Bruhat, A., et al., Amino acids control mammalian gene transcription: activating transcription factor 2 is essential for the amino acid responsiveness of the CHOP promoter. Mol Cell Biol, 2000. 20(19): p. 7192-204.

26. Oyadomari, S. and M. Mori, Roles of CHOP/GADD153 in endoplasmic reticulum stress. Cell Death Differ, 2004. 11(4): p. 381-9.

27. Al-Madhoun, A.S., et al., Phosphorylation of isocarbostyril- and difluorophenyl-nucleoside thymidine mimics by the human deoxynucleoside kinases. Nucleosides Nucleotides Nucleic Acids, 2004. 23(12): p. 1865-74.

28. Al Madhoun, A., et al., Comparative Proteomic Analysis Identifies EphA2 as a Specific Cell Surface Marker for Wharton's Jelly-Derived Mesenchymal Stem Cells. Int J Mol Sci, 2020. 21(17).

29. Bitar, M.S., et al., Hydrogen Sulfide Donor NaHS Improves Metabolism and Reduces Muscle Atrophy in Type 2 Diabetes: Implication for Understanding Sarcopenic Pathophysiology. Oxid Med Cell Longev, 2018. 2018: p. 6825452.

30. Al-Madhoun, A.S., et al., Evaluation of human thymidine kinase 1 substrates as new candidates for boron neutron capture therapy. Cancer Res, 2004. 64(17): p. 6280-6.

31. Al Madhoun, A., et al., Chemically Defined Conditions Mediate an Efficient Induction of Mesodermal Lineage from Human Umbilical Cord- and Bone Marrow- Mesenchymal Stem Cells and Dental Pulp Pluripotent-Like Stem Cells. Cell Reprogram, 2018. 20(1): p. 9-16. 
32. Nunez-Toldra, R., et al., Dental pulp pluripotent-like stem cells (DPPSC), a new stem cell population with chromosomal stability and osteogenic capacity for biomaterials evaluation. BMC Cell Biol, 2017. 18(1): p. 21.

33. Wang, $\mathrm{X}$. and $\mathrm{B}$. Seed, $A$ PCR primer bank for quantitative gene expression analysis. Nucleic Acids Res, 2003. 31(24): p. e154.

34. Abdel-Halim, S.M., et al., Increased Plasma Levels of Adenylate Cyclase 8 and cAMP Are Associated with Obesity and Type 2 Diabetes: Results from a Cross-Sectional Study. Biology (Basel), 2020. 9(9).

35. Fang, Z., et al., ONC201 demonstrates anti-tumorigenic and anti-metastatic activity in uterine serous carcinoma in vitro. Am J Cancer Res, 2018. 8(8): p. 1551-1563.

36. Kanehisa, M., et al., KEGG: new perspectives on genomes, pathways, diseases and drugs. Nucleic Acids Res, 2017. 45(D1): p. D353-D361.

37. Kanehisa, M., Y. Sato, and K. Morishima, BlastKOALA and GhostKOALA: KEGG Tools for Functional Characterization of Genome and Metagenome Sequences. J Mol Biol, 2016. 428(4): p. 726-731.

38. Al-Madhoun, A.S., et al., Detection of an alternatively spliced form of deoxycytidine kinase $m R N A$ in the 2'-2'-difluorodeoxycytidine (gemcitabine)-resistant human ovarian cancer cell line AG6000. Biochem Pharmacol, 2004. 68(4): p. 601-9.

39. Urbanski, L.M., N. Leclair, and O. Anczukow, Alternative-splicing defects in cancer: Splicing regulators and their downstream targets, guiding the way to novel cancer therapeutics. Wiley Interdiscip Rev RNA, 2018. 9(4): p. e1476.

40. Ghigna, C., C. Valacca, and G. Biamonti, Alternative splicing and tumor progression. Curr Genomics, 2008. 9(8): p. 556-70.

41. Siwecka, N., et al., Dual role of Endoplasmic Reticulum Stress-Mediated Unfolded Protein Response Signaling Pathway in Carcinogenesis. Int J Mol Sci, 2019. 20(18).

42. Ma, Y. and L.M. Hendershot, The role of the unfolded protein response in tumour development: friend or foe? Nat Rev Cancer, 2004. 4(12): p. 966-77.

43. Jeng, P.S., et al., BH3-Dependent and Independent Activation of BAX and BAK in Mitochondrial Apoptosis. Curr Opin Physiol, 2018. 3: p. 71-81.

44. Yuan, X., et al., ONC201 activates ER stress to inhibit the growth of triple-negative breast cancer cells. Oncotarget, 2017. 8(13): p. 21626-21638.

45. Ralff, M.D., et al., ONC201: a new treatment option being tested clinically for recurrent glioblastoma. Transl Cancer Res, 2017. 6(Suppl 7): p. S1239-S1243.

46. Infantino, V., et al., A key role of the mitochondrial citrate carrier (SLC25A1) in TNFalphaand IFNgamma-triggered inflammation. Biochim Biophys Acta, 2014. 1839(11): p. 1217-1225.

47. Bartolome, F. and A.Y. Abramov, Measurement of mitochondrial NADH and FAD autofluorescence in live cells. Methods Mol Biol, 2015. 1264: p. 263-70.

48. Ishida, C.T., et al., Metabolic Reprogramming by Dual AKT/ERK Inhibition through Imipridones Elicits Unique Vulnerabilities in Glioblastoma. Clin Cancer Res, 2018. 24(21): p. 5392-5406.

49. Wethmar, K., J.J. Smink, and A. Leutz, Upstream open reading frames: molecular switches in (patho)physiology. Bioessays, 2010. 32(10): p. 885-93. 
50. Balbinot, C., et al., Fine-tuning and autoregulation of the intestinal determinant and tumor suppressor homeobox gene CDX2 by alternative splicing. Cell Death Differ, 2017. 24(12): p. 2173-2186.

51. Yamanaka, R., et al., CCAAT/enhancer binding protein epsilon is preferentially up-regulated during granulocytic differentiation and its functional versatility is determined by alternative use of promoters and differential splicing. Proc Natl Acad Sci U S A, 1997. 94(12): p. 6462-7.

52. Lekstrom-Himes, J.A., The role of C/EBP(epsilon) in the terminal stages of granulocyte differentiation. Stem Cells, 2001. 19(2): p. 125-33.

53. Bedi, R., et al., Human C/EBP-epsilon activator and repressor isoforms differentially reprogram myeloid lineage commitment and differentiation. Blood, 2009. 113(2): p. 317-27.

54. Bedi, R., et al., Human C/EBP-epsilon activator and repressor isoforms differentially reprogram myeloid lineage commitment and differentiation. Blood, 2009. 113(2): p. 317-327.

55. Celia-Terrassa, T. and Y. Kang, Distinctive properties of metastasis-initiating cells. Genes Dev, 2016. 30(8): p. 892-908. 